\title{
Observations of Embryonic Changes in Middle and Late Stages of the Greater Wax Moth, Galleria mellonella (Lepidoptera: Pyralidae)
}

\author{
Muhamad Abidalla1,2, Donatella Battaglia ${ }^{1}$ \\ ${ }^{1}$ Department of Science, University of Basilicata, Potenza, Italy \\ ${ }^{2}$ Department of Biology, Karl-Franzens University of Graz, Graz, Austria \\ Email: muhamad.abidalla@uni-graz.at, mtfallujah13@gmail.com
}

How to cite this paper: Abidalla, M. and Battaglia, D. (2018) Observations of Embryonic Changes in Middle and Late Stages of the Greater Wax Moth, Galleria mellonella (Lepidoptera: Pyralidae). Advances in Entomology, 6, 189-197.

https://doi.org/10.4236/ae.2018.63015

Received: April 25, 2018

Accepted: June 30, 2018

Published: July 3, 2018

Copyright (c) 2018 by authors and Scientific Research Publishing Inc. This work is licensed under the Creative Commons Attribution International License (CC BY 4.0).

http://creativecommons.org/licenses/by/4.0/

\section{(c) (i) Open Access}

\begin{abstract}
The embryogenesis of lepidopteran insects has morphogenetic events accompanying the blastokinesis movements (anatrepsis and katatrepsis) in early and late stages, respectively. Katatrepsis is related to embryonic movement with yolk mass and regression of amnioserosa folds in the second half of the development cycle. The whole mount method and differential interference contrast microscopy (DIC) were used for analysing the embryonic developmental changes. Those changes in the middle and late embryonic periods were described and divided into eight stages: 1) Completion of segmentation and differentiation of cephalic and thoracic appendages (32 - 40 hours post-oviposition h. PO); 2) Expanded growth of cephalo-gnathal and abdominal parts (41 - 60 h. PO); 3) Completion of thoracic appendages and appearance of pleuropodia, katatrepsis (60 - $70 \mathrm{~h} . \mathrm{PO})$; 4) Pre-revolution morphogenetic movement of the cephalo-gnathal region (71 - $80 \mathrm{~h}$. PO); 5) revolution of the embryo (81 - 100 h. PO); 6) Beginning of dorsal closure (101 - 115 h. PO); 7) completion of dorsal closure (116 - $120 \mathrm{~h}$. PO); and 8) full-grown embryo just before hatching (121 - 144 h. PO).
\end{abstract}

\section{Keywords}

Galleria mellonella, Morphogenesis, Katatrepsis, Embryonic Development, Dorsal Closure

\section{Introduction}

The importance of Galleria mellonella was increased with their different uses in different aspects as a parasite of bee hives [1] and a model of pathogenesis studies [2] [3]. Previous studies on the early embryonic stages [4] and postembryo- 
nic development [5] showed there was missed information on the late morphogenesis accompanied katatrepsis movement to the final development of embryos as larvae of $G$. mellonella. Blastokinesis is a movement of the embryo in relation to the yolk mass that consists of two phases of anatrepsis and katatrepsis [6].

Usually, microscopic researches on lepidopteran embryos used the method of sectioned sample that stained with various molecular dyes like Stigmella castanopsiella Kuroko [7], Eriocrania sp. [8], Endoclitasinensis [9], Luehdorfia japonica leech [10], and Endoclita signifier walker [11], while other procedure without sectioning or staining the embryo sample was done as in centipede Scolopocryptops rubiginosus L. Koch [12], and was a succeed method also in this study on $G$. mellonella embryos. The reason to use whole mount procedure without staining is to avoid the difficulties had been occurring with sample preparation in middle and late embryonic stages [4].

The aims of this study were facilitating and providing a microscope data about the characters of late embryonic stages in G. mellonella, especially for improving their results with cryopreservation [13].

\section{Material and Methods}

The reared larvae and microscopic experiments of $G$. mellonella embryos were carried out as joint research with the Centre for Agrobiological and Pedological Research (CREA-Agricultural Research Council, Florence, Italy).

\subsection{Egg/Embryo Collecting and Staging}

Eggs and embryos were obtained from female G. mellonella reared for 6 months at a temperature of $26^{\circ} \mathrm{C} \pm 2^{\circ} \mathrm{C}$ and relative humidity of $70 \% \pm 10 \%$. Eggs were collected on an oviposition paper placed on the top of a cage containing $100-150$ adults with a sex ratio of about 1:1. The oviposition period of females was limited to $30 \mathrm{~min}$. Egg batches were then placed in aerated cages $(10 \times 20 \mathrm{~cm})$ and incubated in a humidified chamber with near-normal honey bee brood nest conditions: Darkness, temperature $30^{\circ} \mathrm{C} \pm 0.5^{\circ} \mathrm{C}$ and relative humidity $85 \% \pm$ $2 \%$. Time zero was taken at the time when fertilized eggs were placed in the chamber. Under these conditions, embryogenesis of $G$. mellonella required 6 days (144 h) until hatching. A total of 180 embryos were obtained for observation of the morphological process, with 10 - 15 embryos being visualized per each interval time of $3 \mathrm{~h}$. During this interval, it could be seen approximately $40 \%-50 \%$ of the embryos with the same embryonic characteristics.

The events and stages of life cycle in $G$. mellonella at $30^{\circ} \mathrm{C}$ were showed in Figure 1 by Abidala [4].

\subsection{Preparing and Visualizing of Embryos}

The procedure steps of sample preparations (dechlorination of chorion and staining by different dyes), microscopic methods and chemicals were described in Abidalla [4]. 


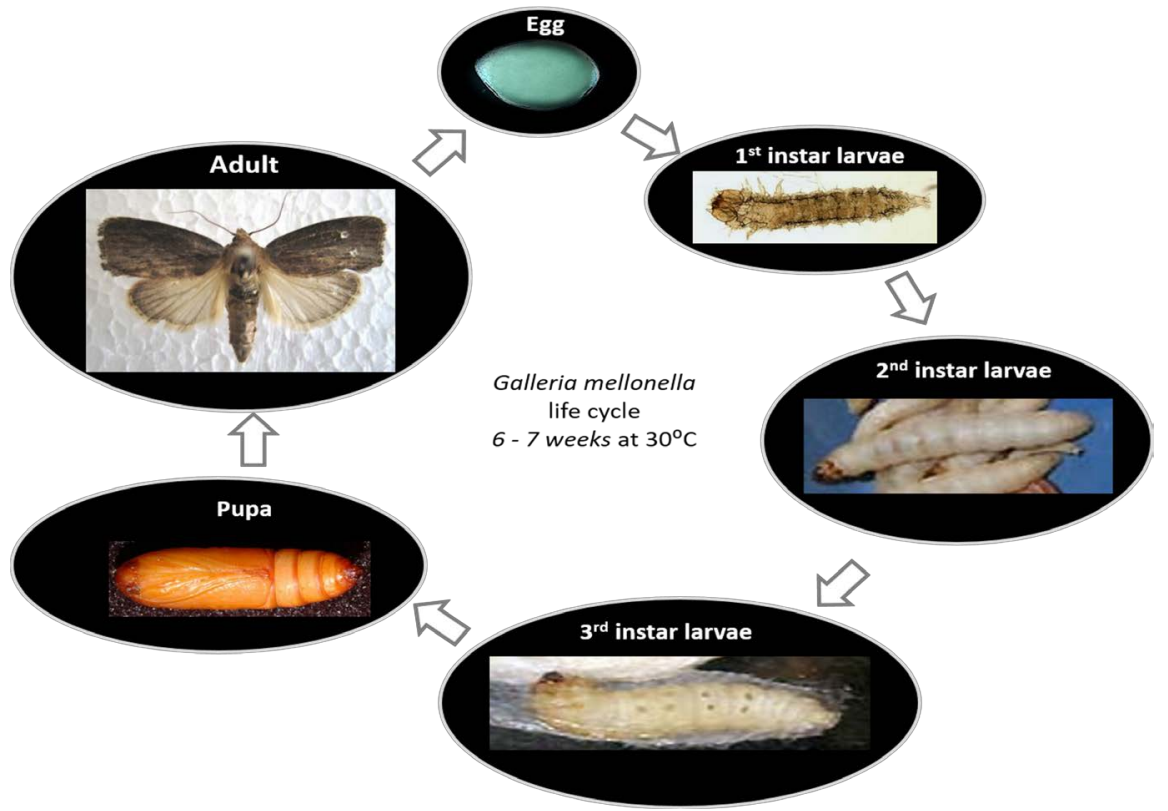

Figure 1. The life cycle events of Galleria mellonella at $30^{\circ} \mathrm{C}$ [4].

The absence of embryonic technologies like time-lapse imaging, and failure of the sectioned sample protocol with $G$. mellonella eggs were motivated reasons to use the whole mount method for observing the morphological changes from germ band segmentations through katatrepsis to the final hatched larvae. The development of serosa and amnion membranes was difficult to imaging in those embryonic stages with method of the whole mount. Previous dyes used in Abidalla work [4], was failed and polarizer-condenser tools played an important role to clarify coloured characters of each embryonic stage under the DIC microscope. The sample procedure of serial dehydration after dechorionation treatments, and fixating in fresh Carnoy's fixative before mounting the sample with glycerol or Canada balsam, were used to prepare embryo for capturing images. The description of embryonic process changes and estimated period of each stage were followed the same classification method for embryonic developmental events and timing by Kobayashi and co-workers on the lepidopteran embryogenesis that distinguish embryonic development markers between each stage sequence [14].

\section{Results and Discussion}

After completion of germ band, embryonic development events of $G$. mellonella from the pre-katatrepsis were described as the following stages:

\subsection{Completion of Segmentation and Differentiation of Cephalic and Thoracic Appendages (Figure 2(A)) (32 - 40 h. PO)}

The elongation of the germ band was followed by different embryonic events including over segmentation, early appendage formation, and early neurogenesis 
as neural groove. The segmentation of Galleria embryos starts in the anterior region, and then proceeds posteriorly. The protocorm differentiates as a segmentation process from its anterior half; hence, the mandibular, maxillary, labial, and three thoracic segments become discernible from the outside. The embryonic membranes of serosa and amnion were supposed to be in the same position mentioned in an early previous development stage, which precedes it [4]. It is one of two development stages pre-katatrepsis.

\subsection{Expanded Growth of Cephalo-Gnathal and Abdominal Parts (Figure 2(B)) (41 - 60 h P0)}

The labral and antennary rudiments appear as paired hemispherical lobes in the protocephalon, while in the gnathal segments, the rudiments of mandibles, maxillae, and labium form three pairs of protrusions. Thereafter, the rudimentary appendages appear in each thoracic segment, but they were missed in the abdominal ones. The neural groove appears along the ventral midline of the embryo. Additional segmentation occurs in the abdominal region and tenth abdominal segment formed the telson. It is last development stage before the embryo starts the katatrepsis.

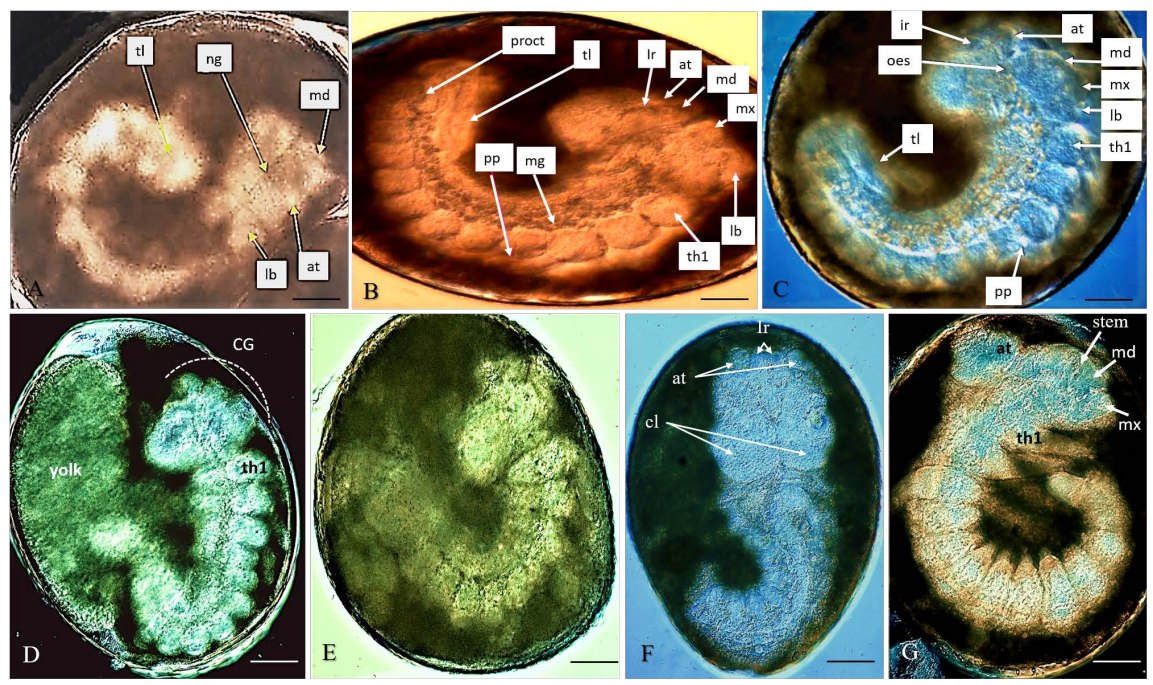

Figure 2. Pre-and post-katatrepsis movement in the embryogenesis of Galleria mellonella. (A) The beginning of appendages formation like antenna (at), labial segment (lb), mandibular segment (md), telson (tl), and the formation of neural grooves (ng); (B) Lateral view of progress development of new appendages like labrum (lr), maxillary segment $(\mathrm{mx})$, pleuropodium (pp), proctodaeum (proct), and prothoracic segment (th1); (C) Longitudinal view of esophageal (oes) development was visualised; (D) Beginning of katatrepsis with moving back the telson and concentrating the amnion-covered yolk in front of doesal side of embryos, as well as to progress cephalo-gnathal (CG) region development; (E) The appendages near telson are turned back with increased size and development progress in the cephalo-gnathal region; (F) Dorsal view of embryo including cephalo lobe (cl), at, and Ir; (G) Completion of katatrepsis movement. Prominences of $\mathrm{mx}$, $\mathrm{md}$ and for the first time showing the stem (primary eyes). The position of three thoracic segments is inside instead of at the peripheral position. Scales $=100 \mu \mathrm{m}$. 


\subsection{Completion of Thoracic Appendages and Appearance of Pleuropodia, Katatrepsis (Figures 2(C)-(F)) (60 - 70 h. PO)}

In this stage, dorsal closure should be occurs within a proposed intact serosa and amnion membranes, which accompanied by a dramatic changes later. The gnathal appendages grow further and the thoracic ones elongate posteriorly. The stomodaeum and proctodaeum develop as long blind tubes. Pleuropodia appear as a pair of small appendage-like projections in the first abdominal segment (Figure 2(C)). In the beginning of the katatrepsis movement in Lepidoptera, usual spherical yolk cells should invade the space between the amnion and serosa in the abdominal region of the embryo (Figure 2(D)). Katatrepsis progresses with the posterior abdominal segments turning ventrally and causing the abdominal end to shift towards the anterior, while the head region turns ventrally (Figure 2(E)). The dorsal side shows advanced development of the cephalo-gnathal region (Figure 2(F)).

\subsection{Pre-Revolution Morphogenetic Movement of the Cephalo-Gnathal Region (Figure 2(G)) (71 - 80 h. P0)}

In this stage, owing to the increasing consumption of the yolk by the embryos, the yolk mass decreases in volume, and finally is left only on the dorsum of the embryo. The embryo assumes a completely superficial position as C-shaped in the lateral view. The morphogenetic movement of the cephalo-gnathal region begins to form the head; the labral rudiments shifts slight posterior ward and makes contact with the mandibular ones. The antennary rudiments also shift to the outer side of the labral ones. Three gnathal segments contrast slightly and fuse to each other. The mandibular rudiments become globose and the maxillary ones are two-segmented and project forwards. The labial rudiments finally move towards the median line and fuse to form a single lobe as will be shown in next stage. The thoracic appendages are fully developed. Pleuropodia still exists although it remains difficult to show it with other parts in images.

\subsection{Revolution of Embryo (Figure 3(A)) (81 - 100 h. P0)}

Revolution refers to the backflip movement at the katatrepsis rotation applied to movements on the longitudinal axis of the egg. In this stage, the posterior abdominal segments are first turned ventrally, so that the abdominal region forms almost a straight line. The abdominal end quickly flexes anterior and finally reaches the level of the prothorax. As the revolution continues, the integration of the cephalo-gnathal region advances, and lastly this region is turned ventrally so that the gnathal appendages surrounding the stomodeal opening project forwards.

At the abdominal end, the tenth abdominal segment and telson fuse together as one; thus abdominal region becomes ten-segmented. New parts here clearly show the brain, midget, oesophageal, protocorm, suboesophageal ganglion, and trachea. 

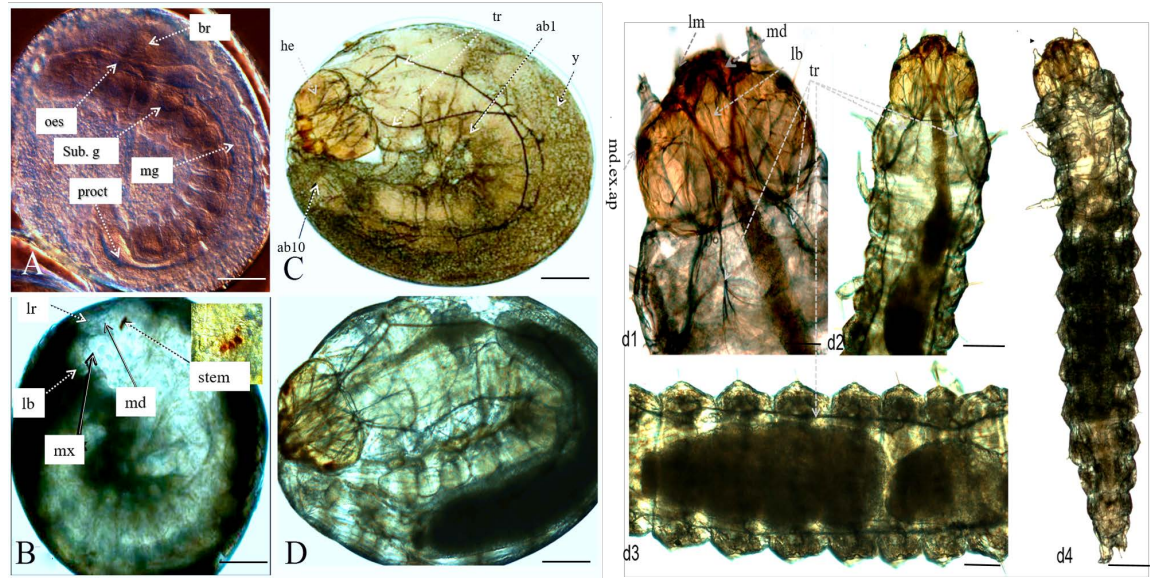

Figure 3. The development progress from revolution stage to hatching larvae in Galleria mellonella embryos. (A) Revolution development process or organogenesis showing the new emerging parts like the brain (br), midget (mg), oesophageal (oes), protocorm (proct), suboesophageal ganglion (Sub. g), and trachea (tr); (B) The advanced mouth formation is more developed and clearly stems as three red points; (C) Completion of dorsal closure process as a function of joint serosa and amnion membranes to cover and pull the rested yolk (y) inside the embryo. In addition, the completion of head (h) formation, three thoracic legs, primary trachea system (tr), and ten abdominal segments (ab1-ab10) are visualised; (D) Pre-hatching larvae stage. d1,d2,d3,d4: Dorsal view post-hatching $G$. mellonella larvae showing completion of head parts as labial (lb), labrum ( $\operatorname{lm})$, mandibular (md), and segments (Maxillary segment (mx) is difficult to show from the dorsal side due to its upper position under $\mathrm{md}$ ), mandibular extensor apodeme (md. ex. ap), and completed trachea system (tr). Scales = A, B, C, D $(100 \mu \mathrm{m}), \mathrm{d} 1(10$ $\mu \mathrm{m}), \mathrm{d} 2$ and $\mathrm{d} 3(50 \mu \mathrm{m}), \mathrm{d} 4(150 \mu \mathrm{m})$.

\subsection{Beginning of Dorsal Closure (Figure 3(B)) (101 - 115 h. P0)}

The katatrepsis movement is responsible for reversing the relative positions of the embryo and yolk. The amnion is replaced by the lateral ectoderm that grows towards the dorsum to later provide the definite dorsal closure. The head is located at the anterior pole of the egg. As the lateral walls grow dorsally in the embryo, these growing lateral walls replace the amnion covering the dorsal surface of the yolk mass. This process of dorsal closure begins first in the posterior region of the abdominal and then proceeds anteriorly. The mouthparts are close to the final shape and completion with advanced development of stem.

\subsection{Completion of Dorsal Closure (Figure 3(C)) (116 - 120 h. P0)}

The dorsal closure progresses and it should be completed when the lateral walls enclose the dorsal region of the mesothorax. Through this process, the basic form of the first-instar larvae is established; that is, the labrum and mandibles become thin, and maxillary and labial ends become sharp and segmented. The antennae changes into very small projections. The thoracic legs are also segmented and pointed. The mouthparts and head have also acquired their larval characteristics. The primary trachea system has clear lines with ramifications. The rest of yolk is placed in the long dorsal side of embryos. 


\subsection{Full-Grown Embryo Just Before Hatching (Figure 3(D), Figures 3(d1)-(d4)) (121 - 144 h. P0)}

At the beginning of this stage, the definitive form of the head capsule is established. At the first, mandibles become sclerotized and pigmented at their distal end. Stemmata or larval ocelli appear as six brown spots on both sides of the head. Then the head capsule and other mouth appendages are also sclerotized. Just before hatching, mandibles usually begin their pulsatile movement, and the full-grown embryo ingests the fragmented embryonic membranes and the shed cuticle through its mouth. The anterior pole of the eggshells (chorion and serosal cuticle) was ruptured by gnawing with the mandibles and the larvae hatches. The larvae immediately eat the eggshells after eating the rested yolk in midget.

The process of gnathal and thoracic segmentation has similar formation process to that in Endoclitasinensis [9], Endoclita signifier [11] and Pieris [15]. The abdomen as in other Lepidoptera insects occurs firstly as a division into eleven segments (ten body segments and a caudal segment called telson). At stage ten, a pair of elevations appears on the ventral side of the caudal segment and the elevations are rudiments of the anal legs. In $G$. mellonella, the tenth abdominal segment does not disappear but remains as in B. mori [16], while in Chilo [17] the ninth and tenth abdominal ganglia unite as one. In addition, the tenth abdominal segment in Antheraea unites with the eleventh segment [18], while the abdominal was 11 segments in moth of Neomicropteryx nipponensis [19].

Dorsal closure in Manducasexta states that is nearly complete when the embryo undergoes the katatrepsis movement [20], while in G. mellonella embryos, the dorsal closure starts during the katatrepsis process.

\section{Conclusion}

The Pyraloidea superfamily is an important species among lepidopteran moths, and the process of development in greater wax moths is mostly similar to the general development in Lepidoptera, with some features that are thought to be characteristic of $G$. mellonella. Each stage has a variable period according to the evolutionary processes' term and histological formation steps. This is an important point to consider when comparing the embryogenesis among lepidopteran embryos. The easy whole mount method could be a successful procedure for fast morphogenesis studying of late stages in insect embryos.

\section{Acknowledgements}

I gratefully thank Prof. Y. Kobayashi (Tokyo Metropolitano University, Japan), Dr. Pio F. Roversi (CREA-ABP, Florence, Italy), Dr. Elena Cosi for their great support to this study. I thank Prof. Christian Sturmbauer (Karl Franzens University of Graz, Austria) for his revisions and comments. This study was partly supported by grants from two projects. The first one is "Storage at low and ultra-low temperatures of entomopathogenic microorganisms, nematodes and 
arthropods important in agricultural defence strategies" from the Agriculture and Forestry Ministry of Italy, and the second one is "Protection of the environment and agriculture against the risk of importation of exotic arthropods" from the National Research Program Prevento of Italy.

\section{References}

[1] Noosidum, A., Hodson, A.K., Lewis, E.E. and Chandrapatya, A. (2010) Characterization of New Entomopathogenic Nematodes from Thailand: Foraging Behavior and Virulence to the Greater Wax Moth, Galleria mellonella L. (Lepidoptera: Pyralidae). Journal of Nematology, 42, 281-291.

https://www.ncbi.nlm.nih.gov/pmc/articles/PMC3380524/

[2] Mylonakis, E., Moreno, R., El Khoury, J.B., Idnurm, A., Heitman, J., Caldernood, S.B., Ausubel, F.M. and Diener, A. (2005) Galleria mellonella as a Model System to Study Cryptococcus Neoformans Pathogenesis. Infection and Immunity, 73, 3842-3850. https://doi.org/10.1128/IAI.73.7.3842-3850.2005

[3] Vogel, H., Altincicek, B., Glöckner, G. and Vilcinskas, A. (2011) A Comprehensive Transcriptome and Immune-Gene Repertoire of the Lepidopteran Model Host Galleria mellonella. BMC Genomics, 12, 308. https://doi.org/10.1186/1471-2164-12-308

[4] Abidalla, M. (2018) Morphogenesis of Early Embryonic Development in the Greater Wax Moth, Galleria mellonella (Lepidoptera: Pyralidae). Journal of Entomology, 15, 1-12. https://scialert.net/abstract/?doi=je.0000.88517.88517 https://doi.org/10.3923/je.2018.1.12

[5] Osinska, H.E. (1981) Ultrastructural Study of the Postembryonic Development of the Neural Lamella of Galleria mellonella L. (Lepidoptera). Cell and Tissue Research, 217, 425-433. https://link.springer.com/article/10.1007/BF00233592 https://doi.org/10.1007/BF00233592

[6] Johannsen, O.A. and Butt, F.H. (1941) Embryology of Insect and Myriapods. McGraw-Hill Book Co., New York.

[7] Kobayashi, Y. (1996) Gross Embryology of a Monotrysia Heteroneuran Moth, Stigmella castanopsiella Kuroko (Nepticulidae, Lepidoptera) and Its Phylogenetic Significance. Transactions of the Lepidopterological Society of Japan, 47, 194-200.

[8] Kobayshi, Y. and Ando, H. (1987) Early Embryonic Development and External Features of Developing Embryos in the Primitive Moth, Eriocrania sp. (Lepidoptera, Eriocraniidae). In: Andō, H., Jura, C. and Jura, C., Eds., Recent Advances in Insect Embryology in Japan and Poland, Arthropodan Embryological Society of Japan, Tokyo, 159-180.

[9] Tanaka, M., Kobayashi, Y. and Ando, H. (1985) Embryonic Development of the Nervous System and Other Ectodermal Derivatives in the Primitive Moth, Endoclitasinensis (Lepidoptera, Hepialidae). In: Ando, H. and Miya, K., Eds., Recent Advances in Insect Embryology in Japan, Arthropodan Embryological Society of Japan, Tokyo, 215-229.

[10] Kobayashi, Y. and Tanaka, M. (1981) Embryonic Development of the Osmeteria of Luehdorfia japonica LEECH (Lepidoptera, Papilionidae). Kontyû (Tokyo), 49, 334-350.

[11] Kobayashi, Y., Tanaka, M. and Miyakawa, K. (1981) Embryonic Development of Alimentary Canal in the Primitive Moth, Endoclita signifier Walker (Lepidoptera, Hepialidae). Kontyû, Tokyo, 49, 641-652.

[12] Sakuma, M. and Machida, R. (2002) Germ Band Formation of a Centipede Scolo- 
pocryptops rubiginosus L. Koch (Chilopoda: Scolopendromorpha). Proceedings of Arthropodan Embryological Society of Japan, Tokyo, 37, 19-23.

[13] Cosi, E., Abidalla, M. and Roversi. P.F. (2010) The Effect of Tween 80 on Eggshell Permeabilization in Galleria mellonella (L.) (Lepidoptera, Pyralidae). Cryoletters, 31, 291-300.

[14] Eastham, L.E.S. (1930) The Embryology of Pieris rapæ-Organogeny. Philosophical Transactions of the Royal Society B, 219, 462-467. https://doi.org/10.1098/rstb.1931.0001

[15] Kobayashi, Y. and Ando, H. (1984) Mesodermal Organogenesis in the Embryo of the Primitive Moth, Neomicropteryx nipponensis Issiki (Lepidoptera, Micropterygidae). Journal of Morphology, 181, 29-47. https://doi.org/10.1002/jmor.1051810105

[16] Nakada, T. (1932) The Resistance of Silkworm Embryo to Abnormal High Temperature and Dryness on the External Morphology of the Embryo. Bulletin of Fukuoka Sericulture Experimental Station, 1, 1-26.

[17] Okada, M. (1960) Embryonic Development of the Rice Stem Borer, Chilo suppressalis. Science Reports of the Tokyo Kyoiku Daigaku, Section B, 9, 243-296.

[18] Saito, S. (1934) A Study on the Development of the Tusser Worm, Antheraea pernyi Guer. Journal of the Faculty of Agriculture, Hokkaido Imperial University, 33, 249-266.

[19] Kobayashi, Y., Tanaka, M. and Ando, H. (2000) Embryology. In: Krestensen, N.P., Ed., Lepidoptera, Moths, and Butterflies, Walter de Gruyter, Vol. 2, Berlin, New York, 494-544.

[20] Konopová, B. and Zrzavý, J. (2005) Ultrastructure, Development, and Homology of Insect Embryonic Cuticles. Journal of Morphology, 264, 339-262.

https://doi.org/10.1002/jmor.10338 